\title{
Virtual Factories with Knowledge-Driven Optimization as a New Research Profile
}

\author{
Amos H.C. $\mathrm{NG}^{1}$ and Sunith BANDARU \\ Production and Automation Engineering, \\ School of Engineering Science, University of Skövde, Sweden
}

\begin{abstract}
This paper conceptually introduces VF-KDO (Virtual Factories with Knowledge-Driven Optimization, a research profile of the University of Skövde, Sweden, which is underway from 2018-2026. The goal of this research profile is to deliver radical innovations in manufacturing research essential to the design and operation of next-generation manufacturing systems. A unique concept proposed in VF-KDO is: knowledge extracted for decision support is achieved through systematically exploring, e.g., using advanced, interactive data analytics techniques on optimal solutions generated via many-objective optimizations on virtual factory models. As the word "driven" means "motivated" or "manipulated", so does KDO have some two-fold meanings: (1) optimizations that aim at generating knowledge, not only mathematically optimal solutions; (2) knowledge-controlled optimizations, instead of some blind/black-box processes. It is this concept of KDO, combining with modular, virtual factory models at different levels, which distinguishes VFKDO from other related research efforts found internationally and in Sweden. The cutting-edge research topics involved in the research profile and their synergy with the digitalization efforts of the 7 partner companies, in form of the development of an intelligent decision support system, can be used to improve the competiveness of the Swedish manufacturing industry by supporting their holistic, optimal and sustainable decision making.
\end{abstract}

Keywords. Industry 4.0, Virtual Manufacturing, Digital Manufacturing, Digital Twins, Simulations, Knowledge Discovery, Data Analytics, Decision Support, Many-Objective Optimization

\section{Introduction}

It is widely acknowledged that manufacturing is the engine of the modern economy, due to its leading contribution to overall productivity and its multiple effects on growth in the rest of the economy. According to the European Competitiveness Report 2013, Towards Knowledge-Driven Re-Industrialization [1], the input from manufacturing to research and innovation is four times higher than its input to GDP. This may explain why the USA and European countries, particularly Germany, have started many initiatives to support their manufacturing industries and strive hard to retain manufacturing jobs [2]. In Sweden, knowing that manufacturing is extremely important for the country's welfare and economic growth, a vision to strengthen its position as one of the world's foremost countries in sustainable manufacturing has been expressed, through various initiatives such as "Made in Sweden 2030" [3] and "Smart Industry" [4], within the last decade.

\footnotetext{
${ }^{1}$ Corresponding Author: amos.ng@his.se
} 
In general, industries today operate on a global market and are subject to a new array of market demands and requirements. The growing environmental awareness drives demand for products with less environmental impact from a life cycle perspective. Governmental initiatives, including environmental regulations and legislation, are putting increasing pressure on industry towards sustainable manufacturing. In addition, societies' higher level of consumer knowledge enhances their demands for new technologies and innovative products. While the development of technology is accelerating at a lightning pace, there is growing global uncertainty, in terms of political and financial instability in various places all over the world, as well as limited access to required resources and unpredictable customer demands. In order to fulfill such demands from customers and authorities, industry is under ever-increasing pressure to be adaptive and agile at all operational levels, from the supply chain, through site, shop, cell to individual process points, with regard to all infra-structural issues concerning equipment, software, and operational principles across these various levels of manufacturing. Therefore, decision makers in industries must consider the strategic, tactical and operational levels, in a holistic manner, during the development of the operations. At the same time, decisions at any level that are solely made on the basis of unit cost and efficiency have ignored the fact that many more criteria are used to judge manufacturing performance [5]. When product variety and volume fluctuation increase, innovative metrics must be developed for sustainable manufacturing systems and to address multiple criteria which include the reduction of the total cost, but simultaneously maximize flexibility, quality, rapid and on-time delivery as well as sustainability [6]. In order to cope with the above-stated tough challenges, digitalization methods and tools supporting the design and improvement of next-generation manufacturing systems must be further developed to consider all operating levels and their complex interactions, in order to provide support for strategic, tactical and operational decision-making within a multi-criteria context.

Industry 4.0, the national strategic agenda of Germany for ensuring the competitive advantage of German industries and transforming the country into a leading nation in the new "industrial revolution", as well as other similar campaigns in other countries, such as the US's "Manufacturing Renaissance", China's "Made in China 2025", all put strong emphasis on the role of digitalization for their future manufacturing industry sectors. "Nyindustrialisering", the equivalent national strategy of Sweden, also strongly emphasizes the crucial role of digitalization for the adaptability and competitiveness of Swedish manufacturing companies. It explicitly identifies "Industry 4.0" as one of the four focus areas for the promotion of Swedish industries to take the lead in digital development and to exploit digitization opportunities. Nevertheless, there is a general misconception that Industry 4.0 is all about digitalization, the Internet of things, Big Data, smart machines, human-robot collaborations and all kinds of advanced manufacturing and their supporting IT technologies. In fact, a careful reading of some definitions of Industry 4.0 [7] reveals that the ultimate goal of Industry 4.0 is actually to "optimize the entire value chain", from gathering customer requirements to the design, manufacturing, deliveries, as well as after-sale services and recycling, in order to improve the overall competitiveness of manufacturing companies. Optimization technologies, therefore, play a significant role in making a modern industry competitive at various levels. As previously mentioned, industries have to deal with several problems that may involve many potentially conflicting objectives. Owing to a large number of intrinsic and external factors that govern these objectives, it is not easy for a company to achieve the best possible configurations. Traditionally, the approach has largely been trial-and-error, 
guided by benchmarking studies and expert opinions at best, and the use of guess work at worst [8][9]. With the advent of cost-effective and easily accessible computing power, industries can now create virtual models for most of its operations. The virtual environment allows simulation models to be built at different levels within a company. The earliest simulation models in the 1960s dealt with inventory management and queuing problems, but over the years, advancements in software technology have enabled the use of virtual models and simulations at supply-chain, factory, line and process levels.

With the constant demands to further reduce production development lead-times, there is huge potential in applying virtual models and simulation technologies to support the design and operation of next-generation factories, as stated in the afore-mentioned "Made in Sweden 2030" report [3]. The use of digital models and simulations for the development of complex products and production systems is well established in Swedish industries, especially within automotive manufacturers. The concept of a virtual factory that can provide the ability to assess performance at multiple levels and generate analytics data, enabling a decision maker to look at the overall factory performance as well as focus attention on analyzing at the equipment/device level has just recently been proposed [10]. Therefore, the term virtual factory, sometimes used interchangeably with virtual copy, digital factory or digital twin can be defined as "an integrated simulation model of major sub-systems in a factory that considers the factory as a whole and provides an advanced decision support capability." [11]. This view of virtual factory that enables overall performance to be analyzed on an integrated simulation model as in a real factory has imposed many technological challenges, including (1) sufficiently highfidelity modeling of individual sub-systems and their inter-relationships, right down to the equipment/device level; (2) ability to generate outputs for the purposes of multicriteria analyses; (3) sub-system models that can be integrated vertically across multiple levels and horizontally with input data sources (e.g. from the real factory) and output data analytics systems.

There are intrinsic relationships among virtual factory, optimization, knowledge and decisions. Central to any decision-making process is the concept of knowledge: the more a decision maker understands the relationship between how to design/run a system and how it performs, the better the decisions that are made [5]. Therefore, the concept of "know why" - to acquire the level of knowledge about the processes at the scientific level with a full model that is able to accurately predict the performance of a design/configuration over a wide range of conditions, represents the core knowledge needed to make optimal decisions [12]. While it seems trivial to relate virtual models with optimization and decision-making, simulation models are in fact evaluative by nature - instead of generating any optimal solutions by themselves, they can only provide performance evaluations of different design alternatives and/or courses of actions. It is when the virtual models are connected to efficient optimization algorithms that optimal solutions can be sought automatically, so-called Simulation-Based Optimization (SBO).

SBO methodologies are capable of effectively searching the vast number of possible solutions and identifying the ones that optimize a given objective. In the presence of more than one objective, multi-objective optimization (MOO) algorithms are used to generate optimal trade-off solutions for the decision maker to choose from [13]. Since most real-world problems, such as production systems development, are inherently multi-criteria by nature and hence inevitably involve multiple optimization objectives [14], these MOO algorithms have been increasingly used in practice during the last two decades. A relatively recent research area connected with MOO is the creation and 
utilization of domain-specific knowledge during runtime. The trade-off optimal solutions generated through MOO may contain hidden clues about what constitutes a good solution. Data mining methods can help reveal such patterns which may ultimately help the decision maker gain a better understanding of the problem. The knowledge gained through data mining can also be used in future related optimization scenarios. Such a process of generating and utilizing the knowledge within $\mathrm{MOO}$ is an active research area at the University of Skövde and has been generally referred to as Knowledge-Driven Optimization (KDO) [15].

Taking into account the vision that competiveness can only be improved with optimized operations, based on knowledge-driven, optimal decisions, as well as bearing in mind all the above-stated challenges. Therefore, the goal of the recently (November 2018) started research profile at the University of Skövde, VF-KDO is to deliver radical innovations in manufacturing research essential to design and operate next-generation manufacturing systems. It will focus on KDO, interactive analytics, as well as multi-level and multi-disciplinary modeling and simulation, including the modeling of humans. The vision of VF-KDO is to enhance the long-term productivity, competitiveness and sustainability of Swedish manufacturing industries, through cutting-edge research and co-productions with seven industrial partners, which is completely in-line with the theme of digitalization for sustainability development promoted in Industry 4.0.

In the remainder of the paper, we first give more details about KDO, particularly a new ontology that supports it. Then a glimpse of how a decision support system (DSS) supporting VF-KDO is provided by outlining its architecture and comprised components, followed by the introduction of an industrial application case and its preliminary results, before the conclusions and future work.

\section{Knowledge-Driven Optimization: beyond Big Data}

The term KDO, as a framework for decision makers to gain knowledge generated by the optimizations and the optimization algorithms, to acquire and incorporate the knowledge and preferences of the decision makers, in order to improve the overall efficiency of any optimizations, was first introduced in [15]. A distinguishing aspect of this research area is that it is knowledge-driven, defined in two ways: (1) perform optimization "driven" by the need to generate knowledge, in terms of rules, patterns, sensitivity and metamodels; (2) gather knowledge that can be utilized to "drive" optimization. The KDO framework has been built to encompass both past studies in this area and future prospective integrations of knowledge and optimization. The KDO framework requires (1) the generation of knowledge from data (often solutions from optimization) and (2) the use of knowledge to drive optimization. These requirements can be met through two closely-related algorithm types, namely, (1) knowledge discovery algorithms and (2) evolutionary algorithms. Knowledge Discovery involves the use of Data Mining (DM) (or machine learning) algorithms that are capable of building knowledge models from optimization data. Most traditional DM methods are not suitable for this purpose, because they treat all features of the data alike. Optimization data is unique, in the sense that it contains both variable values and objective function values, which should be treated in different ways because the variables are what a decision maker can change, while the objective values reflect the consequences of these changes. Decision makers take decisions based on the objective values, hence an appropriate DM technique should be able to extract knowledge about the variables, which is meaningful in terms of the 
objectives. Therefore, existing general-purpose DM methods need to be extended to be used with optimization data. Evolutionary Algorithms are among the most popular optimization methods used in practice. They are based on the principles of natural evolution and known to be effective in solving real-world optimization problems, despite their size, nonlinearities, stochasticity and non-analytic nature. However, the greatest benefit of using evolutionary algorithms rather than other traditional optimization algorithms is their ability to handle multiple optimization objectives. In comparison, emerging approaches with a similar direction that combines data mining with MOO data, sometimes called design space exploration, can be found in [16-17], but their main application is in engineering product design. Other studies that involve the integration of data mining, simulation and optimization can be found in [18-20], however, all of them considered only single-objective optimization problems.

Our research in the area of KDO has progressed in two general directions, offline and online knowledge-driven optimization (see Figure 1). In offline KDO, the knowledge discovery process starts after the optimal solutions are identified. Consequently, the knowledge that is obtained primarily serves to enhance the user's understanding of the problem. Based on the insights gained, the decision maker may choose to rerun the scenario by modifying the original optimization problem. Sometimes, explicit forms of the obtained knowledge can also be stored in a knowledge base and used in combination with an expert system. With the parallel development of competency in the domains of knowledge discovery and evolutionary algorithms, the PAT group is now at a stage where evolutionary algorithms can be improved by using knowledge gained through previous simulations, thus making them very efficient in solving specific problems. This is known as online KDO, which is challenging, both with regard to the knowledge extraction procedure and its integration with the optimization procedure. Significant changes to the optimization algorithm may also be required, in order to incorporate the knowledge into the search process. Three aspects of this integration, which can greatly influence the success of online KDO, are: (1) when knowledge should be extracted, (2) which solutions should be used, and (3) what knowledge should be imposed.

In order to integrate knowledge discovery and evolutionary algorithms into a single framework, it is also important to categorize different types of knowledge. Broadly speaking, knowledge can either be implicit or explicit. Knowledge is said to be implicit when it does not have a specific notation and, hence, cannot be stored and shared easily. On the other hand, explicit knowledge has a predefined format, which makes it easier to codify and, more importantly, to process automatically inside an algorithm. Both implicit and explicit types of knowledge come in many different forms and can, in general, help speed-up optimization, especially in large-scale industrial problems. Some common forms of knowledge that can be extracted from optimization data include, (1) variable and objective correlations; (2) variable importance; (3) metamodels; (4) association rules; (5) mathematical relationships between continuous variables; (6) patterns involving categorical variables, and (7) user preferences. Each form of knowledge requires its own knowledge discovery technique and its own way of integration with an evolutionary algorithm. Here an ontology is used to describe what are the "things" specifically existed within the KDO, based on the above-mentioned concepts, including the relationship between these entities in Figure 2. Such an ontology is incomplete at this stage as the complete ontology for VF-KDO should include entities from the manufacturing and virtual factory domain, which requires more research efforts. 


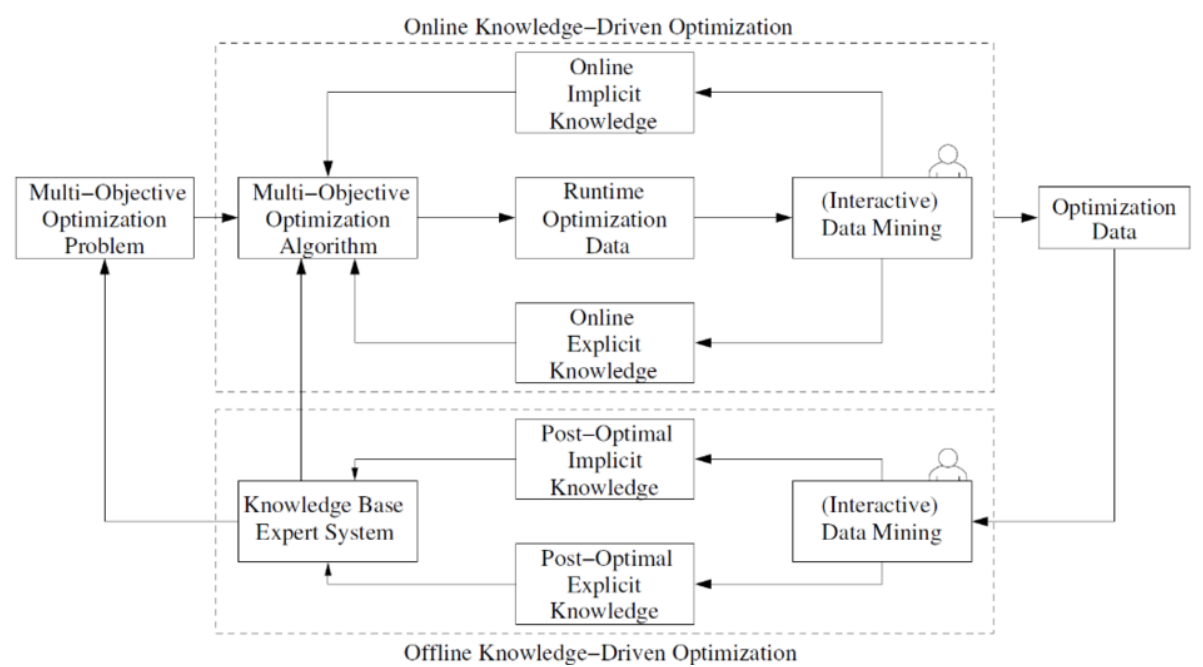

Figure 1. Online and offline KDO, adopted from [15].

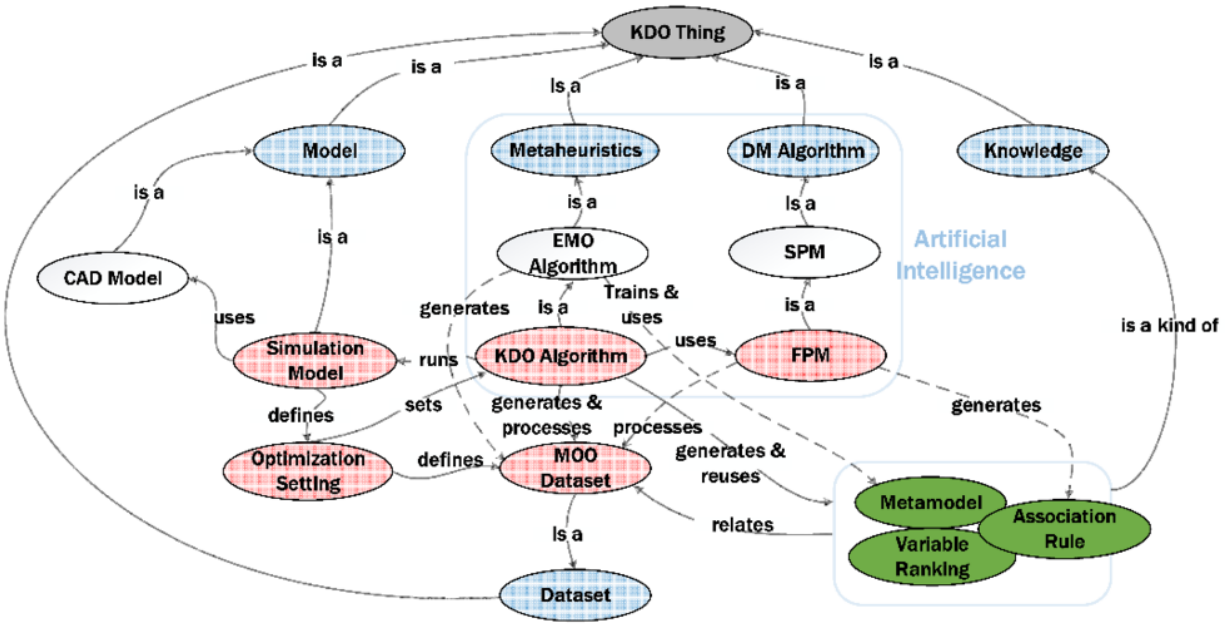

Figure 2. Parts of the Ontology that describe the entities exist for KDO and their relationship.

\section{A VF-KDO based Decision Support System}

With the research outcomes from VF-KDO, we aim at developing a complete integrated framework that combines data digitalization, simulation, $\mathrm{MOO}$, data mining for decision-making support at multiple levels in the industry, in the form of a decision support system (DSS), following the Design Science research methodology [21]. The overall architecture of such a VF-KDO-based DSS is shown in Figure 3. As shown in the figure, multi-disciplinary models for modelling humans (ergonomics), robots, processes (e.g. machining) and systems (e.g., flow simulation) are supported in form of Digital Twins that connect to their real counterparts (i.e. cyber-physical sub-systems). 
One of the most important deliverables of the research in Phase 1 (2018-2022) is, therefore, to supply the data, models and knowledge in the form of modular building blocks, stored in an integrated database system (LINK in Figure 3), for the uses and reuses in various multi-level simulation and optimization scenarios. With these modular virtual models in place and other research outcomes of Phase 1 available, a total of 4 important, long-term, synergy research questions related to the design of a VF-KDObased DSS have to be addressed in Phase 2 of the profile:

- How can the VF-KDO framework be mapped to the decision-making activities occurring in multiple levels of real-world manufacturing companies?

- How can the DSS based on VF-KDO be developed to effectively support the explorations and expressions of the preferences of multiple decision makers at multiple levels in the industry?

- Given that both the SBO and data mining processes behind VF-KDO are highly computationally expensive and significantly more complex with multidisciplinary models, what is the most cost-effective computing platform for the development of a VF-KDO decision support system, in order to support realworld manufacturing applications?

- How can we design a linked data model and knowledge management system that supports the storage, mapping, search and retrieval of factory data, complex models, optimized data and extracted knowledge from multiple levels, in order to make a VF-KDO based DSS feasible for use in a practical industrial environment?

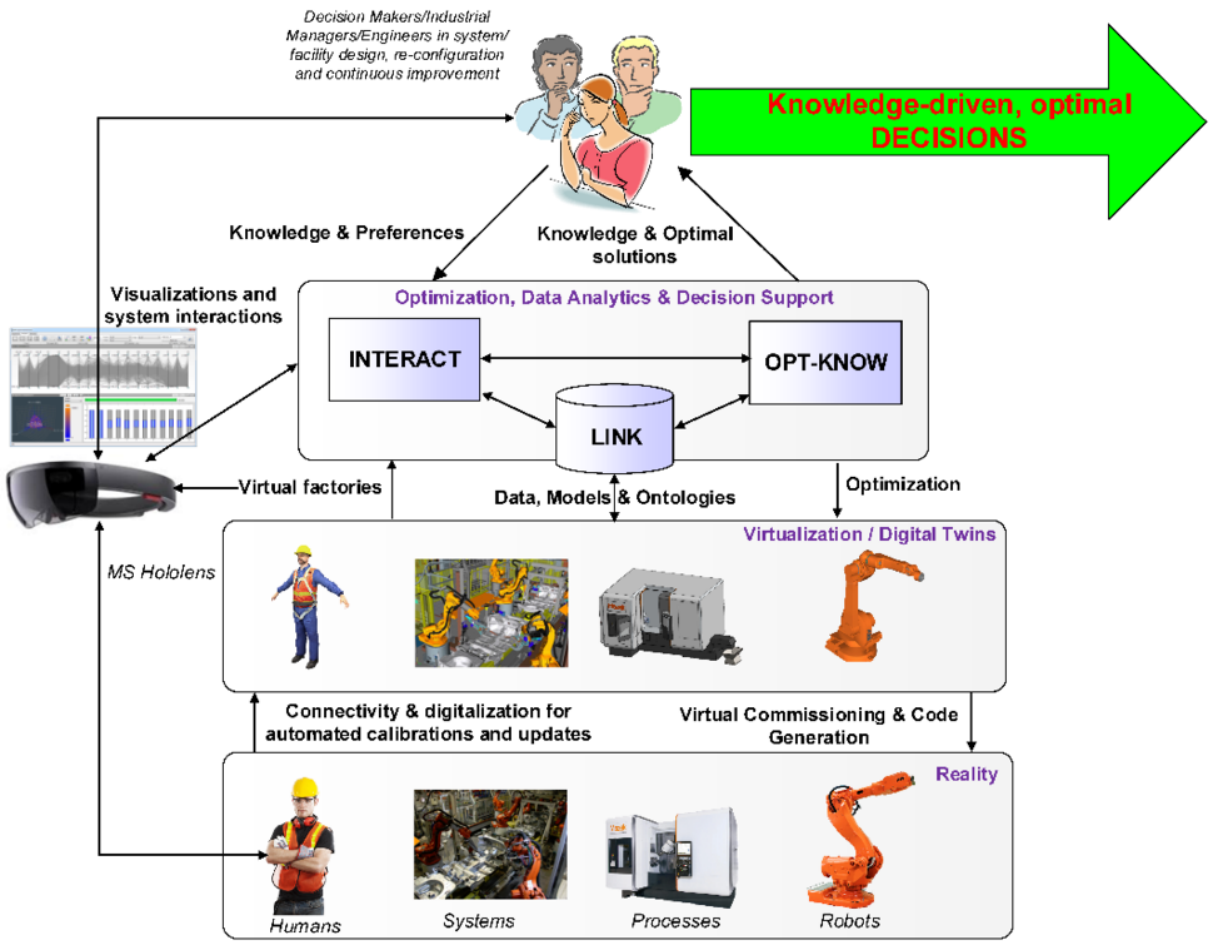

Figure 3. The overall architecture of a VF-KDO based DSS. 
Addressing the above long-term research questions requires not only some multidisciplinary research and industrial teams in simulation modelling but also competences in Artificial Intelligence, Computational Intelligence, general computing and Information System modelling, and above-all Industrial Decision Making. In particular, a relatively unexplored area in the literature and industry is the visualization and interaction models in the support of group decision-making. It is envisaged that VF-KDO will provide a unique way to trace the analysis, visualization and $\mathrm{KDO}$ process of individual stakeholders in a manufacturing system design or improvement scenarios to reveal the rationales of their preferred solutions to each other. Enhancing traceability has been promoted in knowledge sharing for product design [22], but optimization in general but MOO in particular has been rarely considered. In the final section, we use a cycle time and energy consumption trade-off to describe what MOO is crucial in manufacturing system design and configuration.

\section{An Application Study Example}

The concept of Virtual Commissioning (VC) is based on the ability to test and validate systems against a virtual model, instead of an actual, real production system [2223]. The direct and obvious benefits of VC include the reduction in travel time and costs. Furthermore, it also provides a flexible platform that offers the possibility to conduct tests and validations without the stress of real on-site commissioning. Related research efforts on VC applications in Sweden, focused on the generation of control code using formal methods and specific to tending robots of automotive press lines, can be found in Chalmers University of Technology [24] and University West [25], respectively.

Despite the many benefits, VC is still not widely used in automation engineering. Two of the main reasons are the lack of specific knowledge needed to select the right technology and the time-consuming efforts required to develop usable models that represent the geometric, kinetic and dynamic behaviors of the automation devices. Different tools and technologies for VC are available in the market. They use different techniques, such as simulation and emulation, including functionalities focused on different types of automated systems. Industrial automated systems models created using Simulation techniques try to provide a similar behavior of the system. This is usually achieved by using simplified approximations or assumptions, to obtain an idea of how the system reacts and to analyze its outputs. On the other hand, Emulation techniques try to imitate the behavior of a system, in order to perform the same work and produce the same results (2). The emulation model needs the real control software, for example, Programmable Robot Controller (PLC) or robot program, instead of the simulated working sequence. This difference makes emulation a perfect platform not only for $\mathrm{VC}$, but also for the entire life cycle of the system (e.g. maintenance).

Currently, we are extending two emulation packages, namely, Simumatik3D [26] and ABB's RobotStudio to connect to an in-house developed SBO platform [27]. These software packages have been used in different automation courses, including control engineering, PLC/robot programming and system integration. The extension of the software to connect to $\mathrm{MOO} / \mathrm{KDO}$ algorithms is targeted to generate optimization results to determine the optimal trade-off of energy consumption and cycle time when designing a robotic cell. A student project has successfully demonstrated the possibility of energy optimization using this multi-disciplinary optimization concept, by incorporating lowlevel electrical motor models in Modelica [28] and energy consumption calculation from 


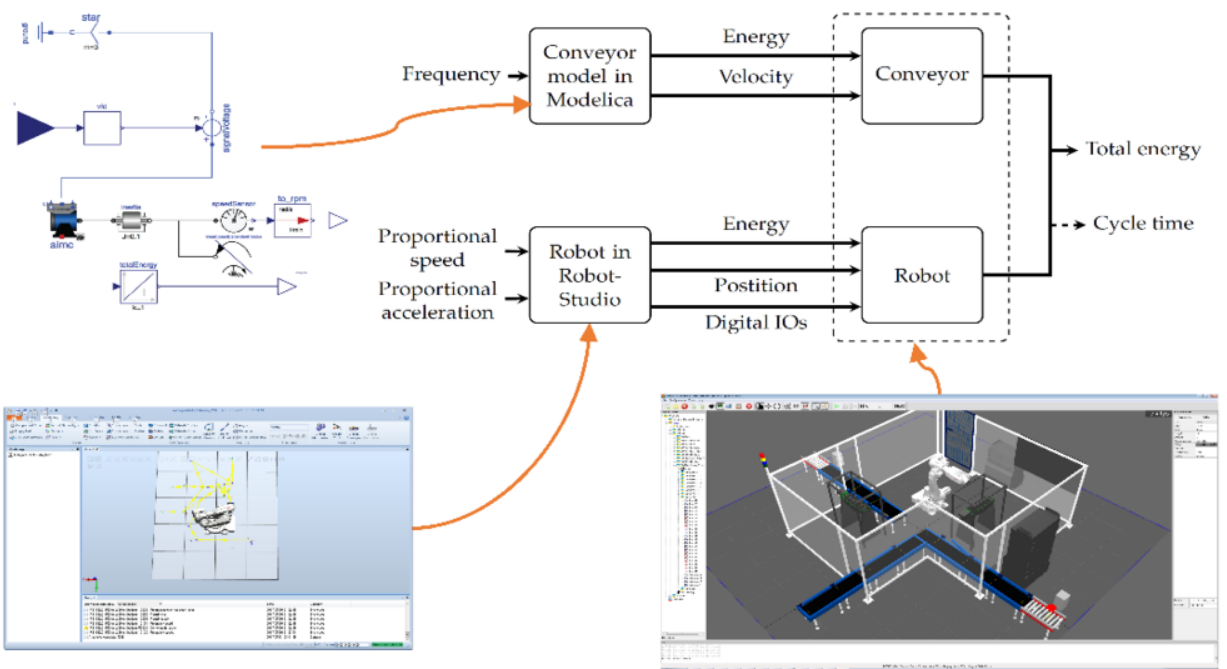

Figure 4. Multi-disciplinary and bi-level models for evaluating energy consumption and cycle time of a robotic production cell [29].

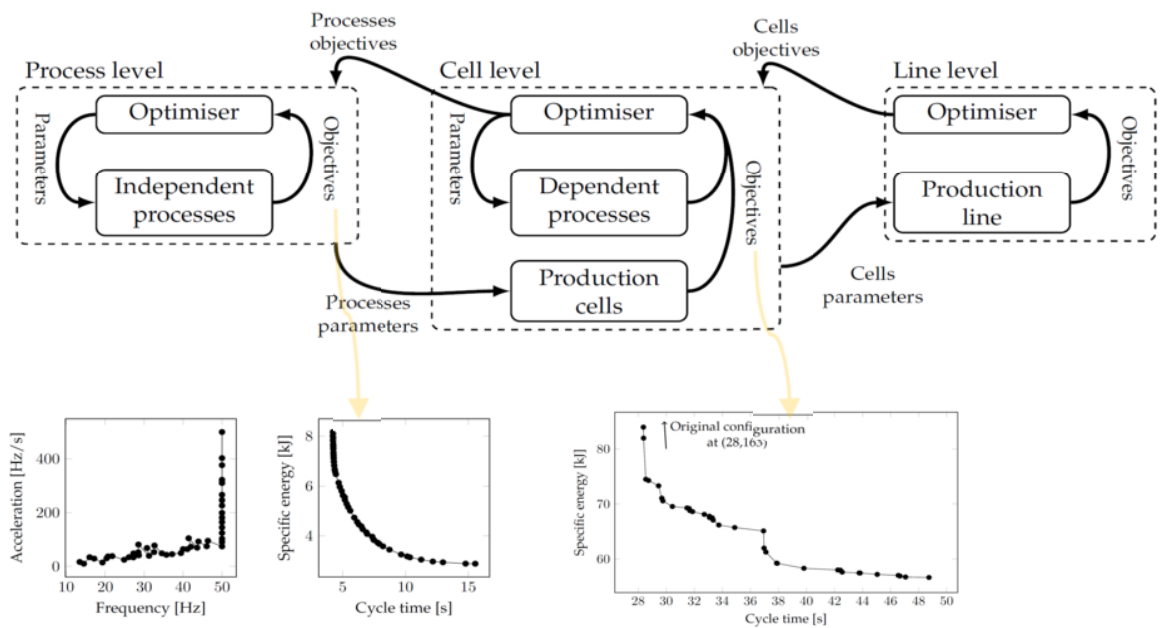

Figure 5. Multi-level optimization with energy consumption and cycle time as objectives [29].

ABB's RobotStudio as shown in Figure 4, within a multi-stage optimization context [29]. The results of the multi-stage MOO allow production engineers to select the most efficient energy consumption for a robot cell in order to achieve the required cycle time (see Figure 5). This successful application study represents a prototype that will be further extended to include not only other automation devices but also human ergonomics model and/or cutting operation models or metamodels from manufacturing processes, so that multiple optimization objectives, related to flexibility, energy, and ergonomics, can be optimized. This type of station optimization, which uses VC tools to connect to real PLC and Robot Control software at the same time, has not previously been attempted. Together with ABB and Volvo GTO, two of the research partners in VF- 
$\mathrm{KDO}$, we are now testing the applications of the same optimization platform to realistic, complex robot cell design scenarios.

\section{Conclusions}

There is a general misconception that Industry 4.0 is all about digitalization technologies and missed that its ultimate goal is actually to "optimize the entire value chain", from gathering customer requirements to the design, manufacturing, deliveries, as well as after-sale services and recycling, in order to improve the overall competitiveness of manufacturing companies. Optimization technologies, therefore, play a significant role in supporting the goal of Industry 4.0. This paper has presented a recently started research profile, called VF-KDO, which is believed to provide a unique framework and a DSS that is able to support industries to make optimized, knowledgedriven decision making for designing and improving next-generation manufacturing systems. This conceptual paper has briefly outlined the research plan and architectural design of such a VF-KDO based DSS that entails multi-disciplinary research topics to be addressed within Artificial Intelligence, Computational Intelligence, Computing and Information System modelling as well as Industrial Decision Making. It is envisaged that synergies from the researchers and industrial companies with various competences, both internally within the consortium and externally with international experts, will be the most important factor that determines the success of this research profile in the coming years.

\section{References}

[1] European Commission, European Competitiveness Report 2013: Towards a Knowledge Driven Reindustrialisation, 2013.

[2] Scientific American, The US could learn from Germany's high-tech manufacturing, October 2012.

[3] VINNOVA, Made in Sweden 2030: Strategic Agenda for Innovation in Production, 2013, https://www.vinnova.se/globalassets/mikrosajter/strategiska-innovationsprogram/agendor/made-insweden-2030-eng.pdf.

[4] Näringsdepartementet, Smart industri - en nyindustrialiseringsstrategi för Sverige, 2015.

[5] N. Slack and M.W. Lewis, Operations strategy, 3rd Edition Prentice Hall, 2011, p. 247.

[6] D.S. Cochran, J.T. Foley and Z. Bi, Use of the manufacturing system design decomposition for comparative analysis and effective design of production systems, International Journal of Production Research, 55(3) (2017), 870-890.

[7] A. Frison, Impact of Industry 4.0 on Lean Methods: and the Business of German and Chinese Manufacturer in China, Kindle Edition, Amazon Digital Services LLC., 2015

[8] G. Chryssolouris, Manufacturing Systems: Theory and Practice, Springer, 2013.

[9] C. Rösiö and J. Bruch, Focusing Early Phases in Production System Design. In Advances in Production Management Systems, Innovative and Knowledge-Based Production Management in a Global-Local World, Berlin Heidelberg: Springer (2014), 100-107.

[10] S. Jain and G. Shao, Virtual Factory Revisited for Manufacturing Data Analytics. In Proceedings of the 2014 Winter Simulation Conference, IEEE (2014), 887-898.

[11] S. Jain, D. Lechevalier, J. Woo and S.J. Shin, Towards a virtual factory prototype. In Proceedings of the 2015 Winter Simulation Conference, IEEE (2015), 2207-2218.

[12] R.E. Bohn, Measuring and managing technical knowledge, Sloan Management Review, Fall (1994).

[13] K. Deb, K. Sindhya and J. Hakanen, Multi-objective optimization. In Decision Sciences: Theory and Practice, CRC Press (2016), 145-184.

[14] A.H.C. Ng, S. Bandaru and M. Frantzén, Innovative Design and Analysis of Production Systems by Multi-objective Optimization and Data Mining. Procedia CIRP, 50 (2016), 665-671. 
[15] S. Bandaru, A.H.C. Ng and K. Deb, Data mining methods for knowledge discovery in multi-objective optimization: Part A - Survey, Expert Systems with Applications, 70 (2017), 139-159.

[16] A. Oyama, T. Nonomura and K. Fujii, Data mining of Pareto-optimal transonic airfoil shapes using proper orthogonal decomposition, Journal of Aircraft, 47(5)(2010), 1756-1762.

[17] L. Graening and B. Sendhoff, Shape mining: A holistic data mining approach for engineering design. Advanced Engineering Informatics, 28(2)(2014) 166-185.

[18] M. Better, F. Glover and M. Laguna, Advances in Analytics: Integrating Dynamic Data Mining with Simulation Optimization. IBM Journal of Research and Development, 51(2007), 477-488.

[19] C. Gröger, F. Niedermann and B. Mitschang, Data Mining-Driven Manufacturing Process Optimization." In Proceedings of the World Congress on Engineering, Vol III, London, U.K, 2012, 4-6.

[20] D. Kibira, Q. Hatim, S. Kumara and G. Shao, Integrating data analytics and simulation methods to support manufacturing decision making. In Proceedings of the 2015 Winter Simulation Conference, IEEE, $2100-$ 2111.

[21] A.R. Hevner, S.T. March, J. Park and S. Ram, S., Design Science in Information Systems Research. MIS Quarterly (2004), 75-105.

[22] M.Z. Ouertani, S. Baïna, L. Gzara and G. Morel, Traceability and management of dispersed product knowledge during design and manufacturing. Computer-Aided Design, 43(5), 2011, 546-562.

[23] P. Hoffmann, R. Schumann, T.M. Maksoud and G.C. Premier, Virtual Commissioning Of Manufacturing Systems: A Review and New Approaches for Simplification. In Proceedings of 24th European Conference on Modelling and Simulation (ECMS) (2010), 175-181.

[24] C.G. Lee and S.C. Park, Survey on the virtual commissioning of manufacturing systems, Journal of Computational Design and Engineering (2014), 213-222.

[25] M. Dahl, K. Bengtsson, P. Bergagård, M. Fabian and P. Falkman, Integrated virtual preparation and commissioning: supporting formal methods during automation systems development. IFACPapersOnLine, 49(12) (2016), 1939-1944.

[26] E. Glorieux, B. Svensson, P. Parthasarathy and F. Danielsson, An energy model for press line tending robots. In 30th European Simulation and Modelling Conference-ESM'2016, October 26-28 (2016), Las Palmas, Gran Canaria, Spain, 1-7.

[27] https://www.simumatik.com/.

[28] P. Fritzson, Principles of object-oriented modeling and simulation with Modelica 3.3: A cyber-physical approach. John Wiley \& Sons, 2014.

[29] I. Exposito and I. Mujika, Reduction of Energy Consumption through Process Optimisation and Variable Production. Final Year Project in Automation Engineering, University of Skövde, 2017. 